\title{
Analysing the Visual Complexity of Web Pages Using Document Structure
}

\author{
Simon Harper* Caroline Jay Eleni Michailidou Huangmao Quan
}

\begin{abstract}
The perception of the visual complexity of World Wide Web (Web) pages is a topic of significant interest. Previous work has examined the relationship between complexity and various aspects of presentation, including font styles, colours and images, but automatically quantifying this dimension of a Web page at the level of the document remains a challenge. In this paper we demonstrate that areas of high complexity can be identified by detecting areas, or 'chunks', of a Web page high in block-level elements. We report a computational algorithm that captures this metric and places Web pages in a sequence that shows an $86 \%$ correlation with the sequences generated through user judgements of complexity. The work shows that structural aspects of a Web page influence how complex a user perceives it to be, and presents a straightforward means of determining complexity through examining the DOM.
\end{abstract}

\section{Keywords}

Visual Perception, Perceived Visual Complexity, Pragmatic Computational Technique, World Wide Web, Block-Level HTML.

\section{Introduction}

Web page designers focus on good visual presentation to implicitly help users navigate, understand, and interact with the content. But current advances in Web page development (such as mash-ups ${ }^{1}$ ) encourage the concatenation of block-level elements dynamically drawn from other sites. Our previous work (see $\S 2.2$ ) has lead us to theorise that this blocky visual chunking (Cascading Style Sheet-CSS, block-level ${ }^{2}$ structure) of Web pages is a major factor in determining perceived complexity (Yesilada et al., 2008). In this case new design practices seem to encourage the perception of visual complexity.

A Web page design is determined by the arrangement of elements or content. It is the 'creative art of executing aesthetic or functional design' (Merriam-Webster, 2006) and it is clear that Web site design can either facilitate or impede a user through the available resources (Holzinger et al., 2009). Recent studies (Ivory and Megraw, 2005; Brown et al., 2012a) of Web site design patterns have found that Web site designs have become increasingly graphical in nature, reliant on browser scripts, and less consistent over time (see $\S 2$ ). Indeed, with the rapid and constant advancement of technology, new ways are constantly being introduced to present information that leads to visually complex Web pages; with significant changes centring around Ajax ${ }^{3}$ Mash-ups, CSS3, Scripts, and eXtensible Hypertext Markup Language (XHTML) / HTML5 coding (Harper and Yesilada, 2010).

Third party work (Hoffos and Sharpless, 1991; Faraday and Sutcliffe, 1998; Lazar et al., 2006; Takagi et al., 2004) shows that distinct layout, smart captions and interesting pictures can transform a wall of dry text into a presentation which users will approach enthusiastically. Such changes may also impact on the perceived visual complexity of a page, however. Although, as we go on to discuss, it is not simply the case that 'lower is better', being able to quantify complexity, and identify the point at which a page becomes overly complex, will help us to construct pages that are both more aesthetically pleasing and more usable.

\footnotetext{
${ }^{*}$ Corresponding Author: School of Computer Science, University of Manchester, Oxford Road, Manchester, M13 9PL - simon.harper@manchester.ac.uk

${ }^{1} \mathrm{~A}$ Mash-up enables the Web developer to pull in content from different sources and facilitate rudimentary orchestration of that content. In this case a pages are often created which are naturally 'blocky'

${ }^{2}$ Most XHTML 1.0 elements permitted within the body are classified as either block-level elements or inline elements. Block-level elements typically contain inline elements and other block-level elements. When rendered visually, block-level elements usually begin on a new line.

${ }^{3}$ asynchronous JavaScript and XML is a group of interrelated web development methods used on the client-side to create interactive web applications.
} 
Previous studies have not investigated ways in which to classify areas of complexity in a replicable way. Indeed, current methods do not provide a universal model for presenting layout complexity in a numeral form. Our work, however, finds that identifying areas of high complexity can be achieved by examining the layout of a page and identifying areas high in block-level elements; and that by using this metric we can place Web pages in a sequence of visual complexity which mirrors the sequence resulting from our previous experiments examining human perception (see $\S 4.5$ ).

Our solution is to analyse the XHTML via the Document Object Model (DOM) conflated with the visually rendered position of each XHTML block-level element. We divide the rendered Web page into a matrix and place the number of block elements whose upper-left corner starts within that cell of the matrix. We are then left with a vector of numbers which acts like a complexity metric of the target Web page. While this matrix is not $100 \%$ accurate, especially as the granularity of the matrix is reduced, it does allow us to automatically place pages into a sequence of visual complexity which shows a correlation with human-sequenced pages (see $\S 3$ ); our theory is therefore supported.

In this way, we contribute to the understanding of Web page complexity and the use of our complexity metric to: (1) Give 'a priori' notice of the likely interaction requirement before a page is encountered (mimicking a visual glance or an element of a glance) (Jay et al., 2006); (2) Give designers the ability to check their pages to help them balance enjoyment with complexity and usability; (3) Identify areas of complexity so that we can build heuristics to drive a transcoding ${ }^{4}$ activity in which complex areas are removed (Harper and Bechhofer, 2007) (see $\S 5$ ); and in the future, (4) assert that there is a relationship between perceived visual complexity and the structural complexity of the page (Yesilada et al., 2008). In this way we hypothesise that complex pages are just as complex to users who cannot see the content - be they visually disabled (Harper et al., 2009) or situationally impaired (Sears et al., 2003) as it is to those who can (see $\S$ 6.1).

\section{Background}

The visual appearance of interactive systems tends to convey more information than we imagine. Contact points that users first make to perceive such a system affect the rest of the interaction process (Lindgaard et al., 2006). The perceived complexity of a scene can have a significant physiological impact. Tuch et al. (Tuch et al., 2009, 2011) demonstrate that the visual complexity of Web sites has multiple effects on human cognition and emotion, impacting upon experienced pleasure and arousal, facial expression, autonomic nervous system activation, task performance, and memory. The complexity of information also affects how it should be delivered via the Web (A. Holzinger and and Albert, 2008). In this section we provide an overview of the visual characteristics shown to influence judgements of complexity, and consider predictive models, before describing our previous work examining the determinants of Web page complexity.

\subsection{Visual Complexity}

Complexity can be defined as "the degree of difficulty in providing a verbal description of an image" (Heaps and Handel, 1999; Oliva et al., 2004). People are able to form judgements about a stimulus in less than $50 \mathrm{~ms}$, and do this through the perception of the low spatial frequencies of a scene (Bar et al., 2006) or Web page (Thielsch and Hirschfeld, 2012). Textures with repetitive and uniform oriented patterns are regarded as less complex than disorganised ones. A visual pattern is also described as complex if its parts are difficult to identify and separate from each other (Oliva et al., 2004). Perception of image complexity depends on the extent to which objects are grouped, the quantity of the parts an observer perceives in the scene, familiarity with the scene and existing knowledge of objects inside the scene. Visual complexity is mainly represented by the perceptual dimensions of quantity of objects, clutter, openness, symmetry, organisation, and variety of colours (Rayner, 1998; Oliva et al., 2004). Studies also report a positive relation between visual complexity and the computer-generated bitmap size (Donderi, 2006), or jpeg size (Tuch et al., 2009) of an image of a Web page, as well as with the emotional and psychophysiological effect of the image (Tuch et al., 2009).

The visual perception of Web sites is affected by cognition, content and form (Germonprez and Zigurs, 2003). Human cognition affects how a user retrieves and uses information in a Web site. Content on the

\footnotetext{
${ }^{4}$ Transcoding is the process of transforming the representation of the Web page into another format, and is based on reverse engineering and design rediscovery techniques.
} 
Web site and the amount of information that is available affects complexity since it can cause information overload on a page. The form of the Web site with respect to user interface, navigation, and structure is a further factor that affects page complexity.

Aesthetic pleasure has been attributed to 'processing fluency': the ease with which users can process interface stimuli (Reber et al., 2004). This theory indicates that simple layouts should be processed more fluently, and therefore appraised more positively. Studies confirm that such a link between simplicity and aesthetic pleasure (Choi and Lee, 2012) and simplicity and usability (Moshagen and Thielsch, 2010) does indeed exist. Tuch et al. (Tuch et al., 2009) presented 36 homepage screenshots to 48 participants in a laboratory setting where the screenshots were of consistent dimensions and there was no interactivity. Using various affective measures, as well as self assessment regarding arousal and valence, they found that the lower the complexity the higher the pleasure, the lower the pleasure the higher the complexity. Other work, including our own, finds an inverted 'U-shaped' relationship (Berlyne, 1960, 1974; Leder et al., 2004; Harper et al., 2009), indicating that people prefer to experience a moderate level of complexity, rather than very high or low levels. Pandir and Knight (2006) (Pandir and Knight, 2006) suggest complexity is not a direct predictor of pleasure, while interestingness is ${ }^{5}$.

Ivory et al. performed a quantitative analysis of Web page attributes (e.g. number of fonts, images, and words) using a large collection of Web sites (Ivory et al., 2000, 2001). The Web sites were judged based on content, structure and navigation, visual design, functionality, interactivity, and overall experience and were selected from categories such as finance, education, community, health, service and living. They found that "Web pages and sites differ from each other on many dimensions, such as layout quality, screen coverage and information quality" (Ivory et al., 2000). Also, in the same study, metrics concerning page composition, such as word and link count, page formatting, such as emphasized text and text positioning, along with overall page characteristics, such as page size, helped to predict (with $63 \%$ accuracy) the ranking a page received from human judges. Using the first study and the same characteristics of the page-level elements, they developed profiles of the Web pages based on the overall ratings that distinguished between good and bad pages with respect to design and usability (Ivory et al., 2001). The above studies (Ivory et al., 2000, 2001; Ivory and Megraw, 2005) were part of a project that tried to develop techniques to empirically investigate all aspects of Web site design, but did not attempt to define and investigate visual complexity. Our empirical work (Michailidou, 2005) using the above characteristics and results focused on Web page design and the structure that determines visually simple and complex designs. Developing a framework with the help of the Web page's elements such as paragraphs, tables, lists, and menus, helped to identify the main structural factors that cause visual complexity. It indicated that the visual complexity of Web pages depends on the presentation of the page's elements, as well as the density and diversity of the elements that are presented, and that these aspects also overlap with visual aesthetics. We found that users classify visually complex Web pages using terms such as 'difficult', 'complicated', 'inaccessible', 'scary', and 'ugly', while they classify very simple Web pages as 'dull', 'boring', 'unappealing', 'amateur'. The best Web sites we have found are those in the middle which seem to hit the 'sweet spot' of being visually complex enough to maintain interest and attract users, while being visually simple enough to facilitate searching and browsing.

Altaboli and Lin (2011) proposed a regression model predicting the effect of balance, unity and sequence of screen layout elements on perceived interface aesthetics. There was a positive relationship between unity the extent to which visual objects on the screen seems to belong together as one object - sequence - the extent to which objects are arranged in a sequence that facilitates the eye movements made during reading - and simplicity.

Stickel et al. (2010) modelled complexity as a function of the number of functional elements (buttons, drop boxes etc.), organisational elements (binding boxes, pictures etc.) and the summed entropy of RGB values (calculated using MatLab script) on a Web page. The model was shown to correlate strongly with user ratings of page complexity, as well as the compressed file size of the page image (Donderi, 2006; Tuch et al., 2009).

Whilst these studies have shown that it is possible to predict page complexity in a number of ways, calcu-

\footnotetext{
${ }^{5}$ Pandir and Knight, however choose Flash based sites from Macromedia's 'Site of the Day' but give no other rational than that, as to assumptions of visual complexity.
} 
lating the metrics for comparison has involved abstracting the Web page - in particular turning it into an image - rather than using the underlying page code. The work reported in the rest of the paper indicates that this abstraction may not be necessary, as complexity could be determined by considering the document structure of a virtually rendered page.

\subsection{Determining Web page complexity through card sorting and triadic elicitation}

It is important to view this related work, and the new work described here, in the light of our previous investigations (Harper et al., 2009), which were undertaken to understand the way in which users interact with Web resources, and classify those resources based on perceived visual complexity. This allowed us to understand if visual complexity was synonymous with the more general complexity of Web pages.

Our initial empirical experiment ${ }^{6}$ used card sorting and triadic elicitation to build an initial baseline sequence of 20 Web pages, and provide an understanding of what aspects of the pages' layout and presentation underpinned the complexity judgements. The pages where chosen based on their visual appearance, research undertaken by IBM (Amitay et al., 2003), and their Web ranking from 'Alexa'7. Twelve participants (6 male and 6 female) took part in the experiment on an individual basis.

In the first part of the study participants were shown hard copies of the pages and asked to rank them in order of complexity. This ranking formed the basis for comparison with the algorithm reported in this paper.

Following the ranking activity, participants were asked to place the pages into piles of low, medium or high complexity, and explain the reasons for their decisions. Finally three pages were chosen at random, and participants were asked to say which two pages were most similar, and how they were different from the third page. This process was repeated three times. Our analysis showed that objects that make pages more complex are not necessarily highly visual components, but those which signify the possibility of increased cognitive load.

We initially analysed document components such as: (1) The block-level elements - this helps us assess the visual rendering of the grid structure; (2) The number of words per block-level element - to assess the context switching required by the user; (3) The colours of each block as they relate to the containing block - this allows us to see if there are large colour changes; (4) The number of images and animations along with their position and size; (5) The number of forms present; and finally (6) The number of links along with their visual rendering - links with different colours to the surrounding text increase the page complexity to a greater extent than those that have the same colour.

Given these six factors, the most important for perceived visual complexity was the visual representation of XHTML block-level elements. In this case our continuation work (explained next) proposes an algorithmic method to identify visual complexity through an analysis of these block-level structural elements. We hypothesise that visual complexity, expressed only as block-level elements within the DOM, is an indicator of perceived complexity, and as such that the ranking generated by our algorithm will correlate with that generated by users in our earlier study.

\section{Technical Approach}

Our algorithm (Listing 1) maps the Web page into a complexity ranking using a matrix overlaid over the content. Initially, the Web page is rendered and the position of each element is fixed. The algorithm was designed to work with 'standard' Web pages between $760 \& 990$ pixels wide. Indeed, the pages we selected were not 'responsive' 8 being set to a maximum of 1000 pixels wide and adding white space as the user agent viewport increased. In this case, testing at higher resolutions was both ineffectual and limited by the 'Flying Saucer' server side renderer ${ }^{9}$ which builds the rendering based upon the Web site specification. In the next step, the matrix of the page is extracted and all features of the block level elements are converted

\footnotetext{
${ }^{6}$ Full methodology and materials are available to allow third-parties to confirm our experiments http://wel.cs.manchester . ac.uk/research/vicram/studies/metrics-study and as a technical report (Michailidou and Harper, 2006).

${ }^{7}$ http://www.alexa.com/

${ }^{8}$ http://en.wikipedia.org/wiki/Responsive_Web_Design.

${ }^{9}$ Flying Saucer Project Web Site http://code.google.com/p/flying-saucer/ viewed in August 2011.
} 
to numbers. For this experiment, our model only extracts the number of block-level elements, and no other visual features. We then associate each element with its grid position. Each cell of the matrix is checked to determine if any part is next to a border and if the cell is not full size it is truncated. If the grid cell is at a border and is truncated, we simply delete it from the vector analysis. Then the number of block level elements in each cell is calculated. Finally we consider the element density and distribution together. The algorithm ranks the Web pages according to the sums of their average and deviation (see Figure 1c); in this way page features are associated with grid cell positions and therefore block-level element density and diversity can be associated with the numerical index of the grid. This cell indexing approach provides a way of specifying and analysing Web pages in detail resulting in a better characterisation of each Web page. Therefore, if we know the grid location of the starting point of each block-level element (outlined in Figure 1a) we can create a vector. In this case, we analyse the XHTML along with the DOM conflated with the visually rendered position of each XHTML block-level element (see Pseudo-Code Listing 1). We divide the rendered Web page into a matrix and place the number of block elements whose upper-left corner starts within that cell. We are then left with a vector of numbers which acts as a complexity metric of the target Web page. Because the density of the block-level structures can now be evaluated by a matrix average, and a deviation value can be obtained by analysing the metric, we can arrive at a decision as to the complexity of the page. We can also adjust the model granularity by changing the size of the grid; a smaller grid size can catch more details of the layout structure. However, in any comparisons the same grid size must be used. The three main steps for encoding layout by using this grid based approach are:

1. Find all of the elements associated with visual complexity (outlined in Figure 1a). In our research, for practical reasons, we consider block-level elements only. Because in the XHTML specification, most block-level elements are visible, they are therefore convenient to find using a DOM parser. Then the information regarding positions and extents of each element are extracted (the block-level elements' positions are represented by the coordinates of their top-left corner);

2. Build a grid which overlays the Web page (see Figure 1b). The size of each cell is decided in order to catch sufficient details and avoid redundant calculations. In our case, screen resolutions are fixed at a maximum width of 1000 pixels and most visible block elements are bigger than 50 pixels both in height or width, our grid's size is $200 \times 200$ pixels. Grid sizes from 50 to 300 pixels were systematically trialled, and 200 was chosen because it provided the most optimal results. By 'optimal' we mean that the automated ranking provided the best fit to the manual ranking. The large size for each cell caught more global layout features than regional ones; and finally,

3. Map the features of elements in each grid into the grid numerical values thereby creating a vector (see Figure 1c). Other features can be added into this matrix only if they can be represented numerically. For example, font styles can be represented as numbers reflecting their visual complexity, and colour scheme complexity can be represented as numbers according to overall contrast of colours in the grid.

The stages above describe our complete methodology, however, our algorithm does not include aspects such as font styles, colours, images etc ${ }^{10}$. Although our complexity model takes these attributes into account, analysis of these factors have not been included in the programmatic tool, because their calculation increases the time taken to output the metric's result beyond an acceptable level. Indeed, our work is different in that we only use block-level elements ${ }^{11}$ which are typically represented by the whitespace forming edges around a specific area. The main predicted failing with this approach is that the users must perceive there to be a block present which may not happen for two reasons: firstly our metric does not take colour changes into account, which are important if elements are nested within each other; and secondly because a block-level element can be styled to be inline in the visual rendering. However, our approach is pragmatic in that an analysis of block-level elements within the DOM is computationally possible and returns results at a very high speed.

\footnotetext{
${ }^{10}$ Indeed, these have already been used as metrics by previous researchers (see $\S 2$ ), their results being widely known.

${ }^{11}$ Block-level elements typically contain inline elements and other block-level elements. When rendered visually, block-level elements usually begin on a new line (examples are divs, headings, iFrames, paragraphs etc.).
} 


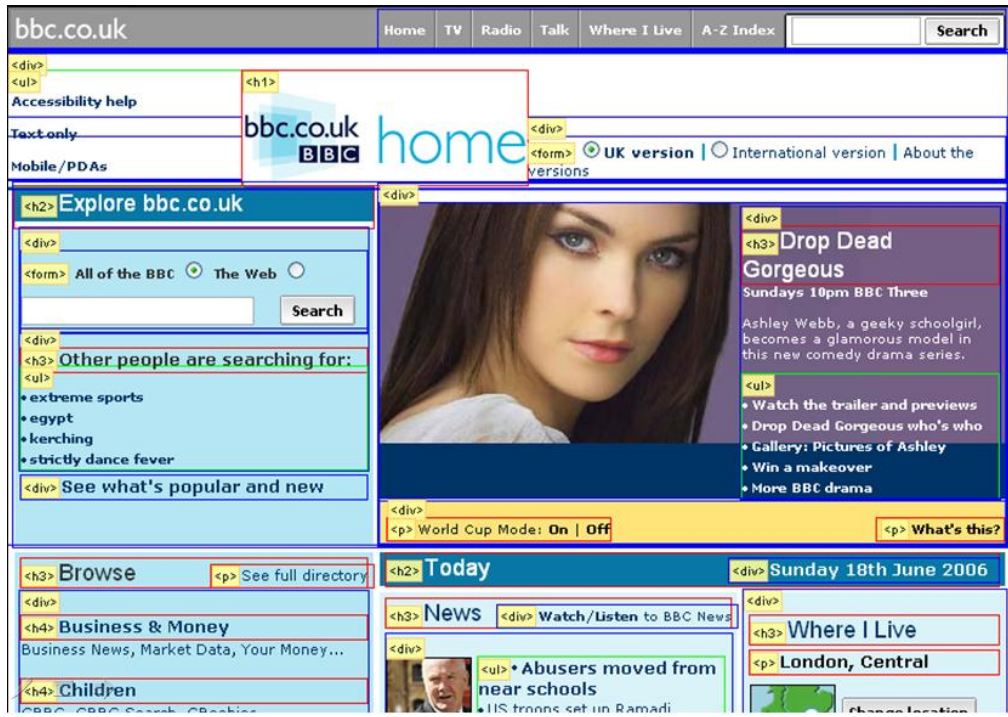

(a) Block-level Elements Outlined and Labelled

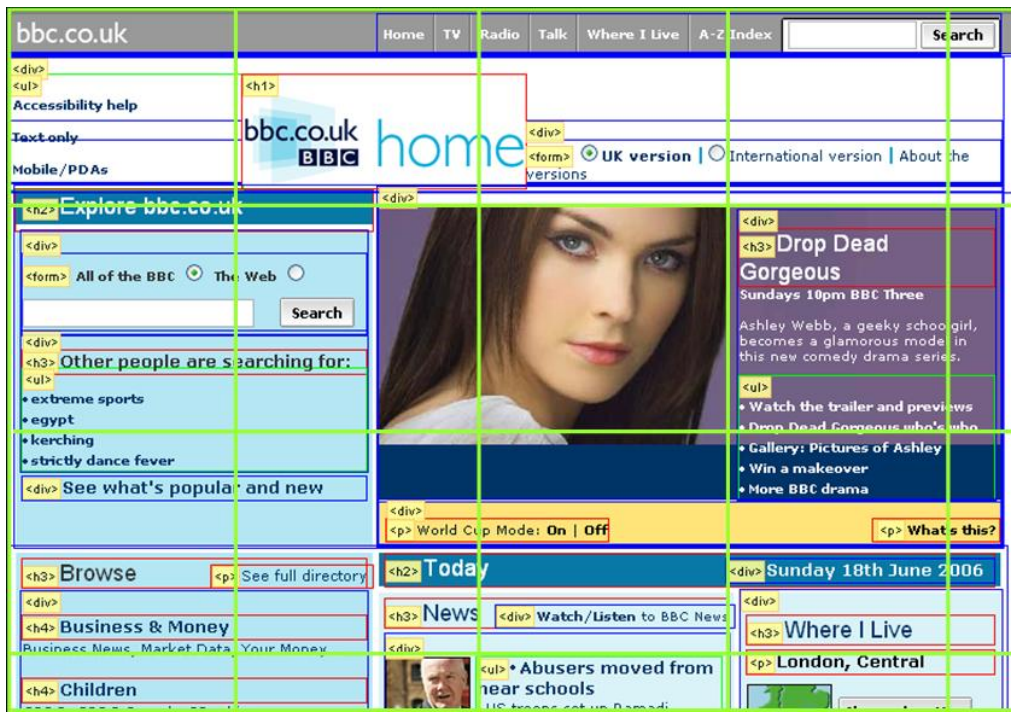

(b) We Can Find All block-level Elements Located in a Certain Grid

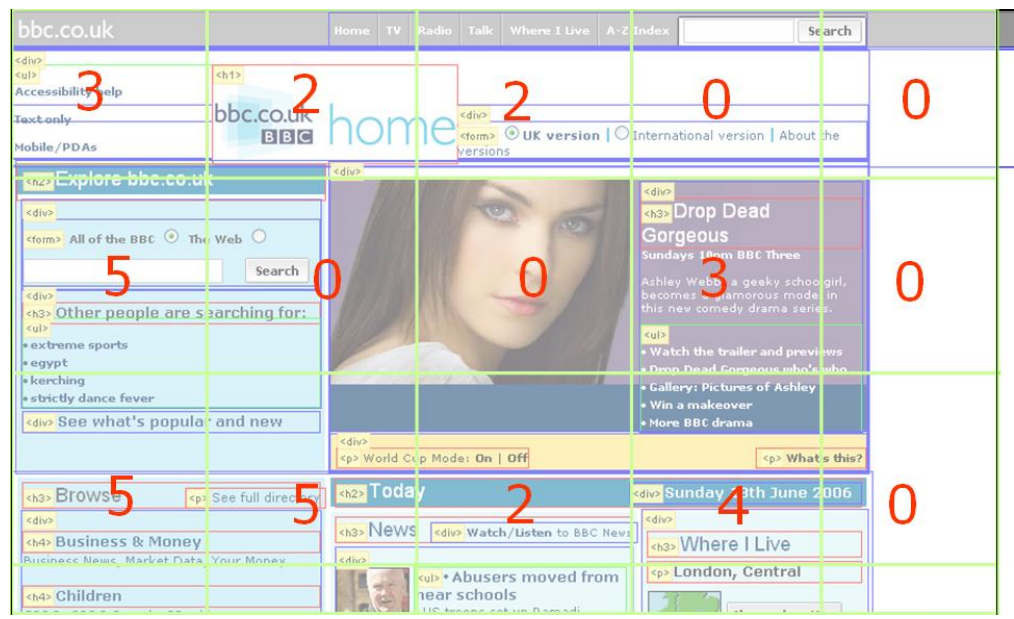

(c) An Example of Grid-Base Method to Encode Layout Metrics

Figure 1: Identifying block-level Elements 
Our experimental system takes any kind of HTML page and passes it through 'Tidy'12 to produce a clean and compliant XHTML page. We then use the Flying Saucer system to generate a virtual rendering of the page on a 'Tomcat' ${ }^{13}$ server. We virtually segment the page and analyse the block-level element positions based on our algorithm and by stepping through the DOM and CSS Application Programming Interface (API) in concert. Our method then outputs the complexity matrix for results prediction. In this way, blocklevel element density and diversity can be associated with a numerical matrix of the grids; a vector is then created. The cell-feature association provides a way of specifying and analysing Web page characteristics in detail, resulting in a complexity metric of each Web page. Additionally, this algorithm is flexible and extendable and means that we can calculate our complexity metric when pages become available, to both refine and enhance our models.

As stated, we have found that some of the most important factors for visual complexity are the structural properties of a page. As we know, XHTML pages contain structural elements that are often used by authors to better structure content. However, parsing XHTML to obtain a proper representation (e.g., DOM Tree) of the structure of the document itself represents a major challenge. The main problem is that XHTML does not enforce a strict syntax and grammar. Creating valid code is necessary for DOM parsing because the parser can only work on an XML or XHTML input stream, and the input Web page must be validated with no errors. Fortunately new XHTML rendering engines (e.g., Gecko ${ }^{14}$ ) overcome these difficulties by making assumptions regarding the badly formed underlying code; the so-called 'quirks mode'. Quirks mode makes browser specific repairs to badly formed code, and these repairs are therefore inconsistent between browsers. In this case a consistent solution is required which can both find and fix errors in the markup language source code. HTML Tidy is a tool that can clean up the underlying code of a Web document. It is a command line tool that takes an HTML file and generates a new XHTML compliant file with cleaner code; importantly the output is browser independent.

Listing 1: Pseudo-Code Listing for the Classification Algorithm

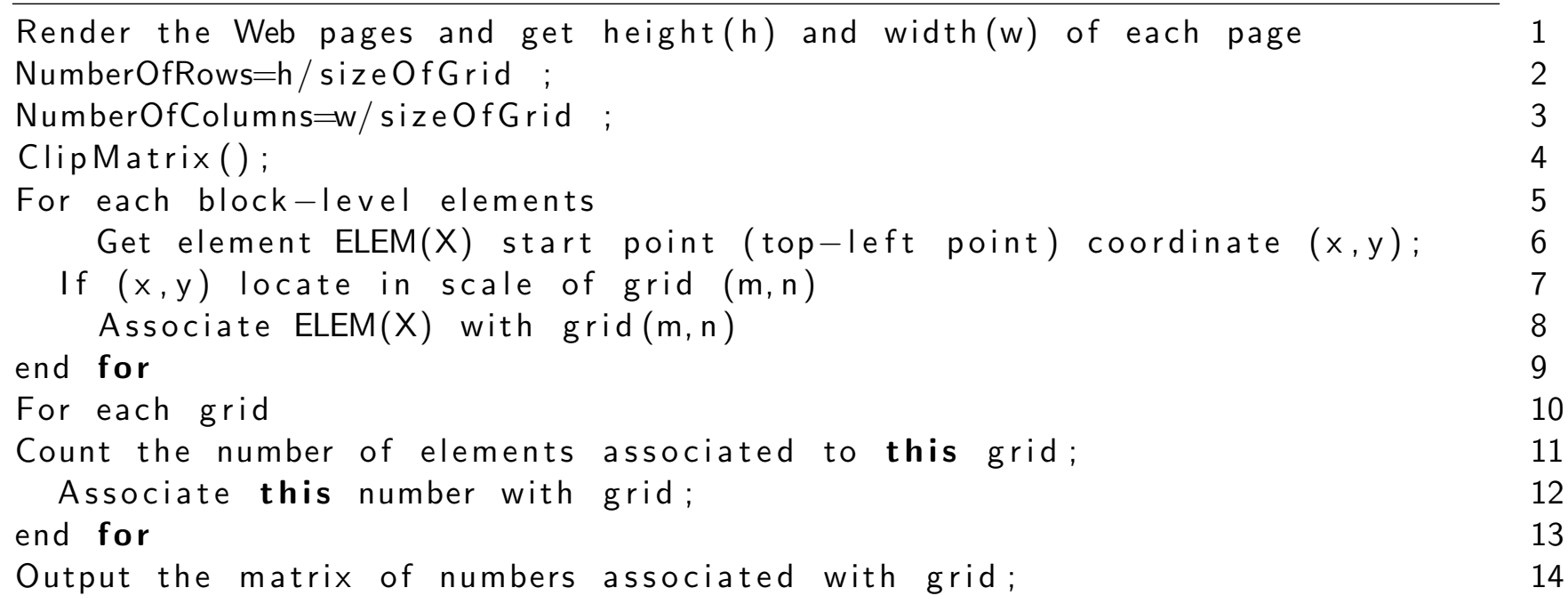

Our system uses a DOM parser for block calculation and we use the Flying Saucer Java API - a stand alone renderer that does not require browser support - to perform these calculations. While Flying Saucer is suitable for server side proxy configurations, it is limited in its support for Javascript, 'Flash', Scalable Vector Graphics (SVG), or legacy HTML. However, we consider support for server side deployment to be of more benefit for a prototypical experiment than support for non-standard languages. Indeed, the rendering

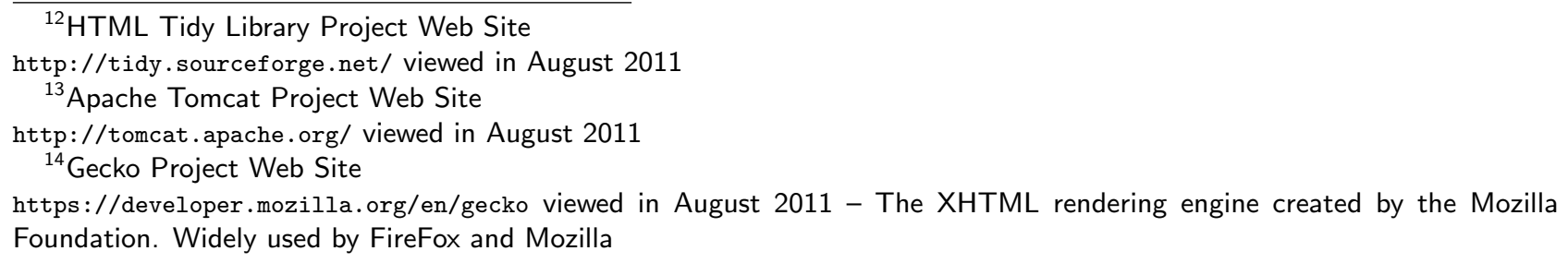


does occur on the server correctly with the image, iFrame, Flash, and SVG extents taken into account and so in these cases our algorithm is unaffected; another benefit of only looking for block-level elements.

In effect we are using DOM block-level elements as a proxy measure for visual complexity. Therefore, our evaluation (see $\S 4$ ) compares our previously ranked pages with those ranked by our algorithm to decide if this proxy measure really is appropriate and accurate.

\section{Evaluation}

Our computational algorithm allowed us to automatically derive a complexity rank for each of the pages previously ranked by hand. We could then order these and compare that order to the manual baseline hand ranking. In this case we use a Spearman correlation analysis as well as a Kendall tau test. $\tau$, is a non-parametric correlation test, and is usually used rather than Spearman's correlation test when there is a small data set with a large number of tied ranks. In addition to comparing the manual ranking with our computational ranking, we also compare it with the file size of the jpeg images of each Web page, which has previously been demonstrated to correlate with perceived visual complexity (Tuch et al., 2009).

\subsection{Population and Sampling}

The population for this experiment is the sample of Web pages previously drawn in our initial experimentation. The pages were selected to be representative of sectors such as public information, business, academia, entertainment and leisure, Web services such as search engines and personal home pages. A full list and screenshots of the pages are provided on the study Web site ${ }^{15}$. All pages are used except S02 as this page was completely redeveloped in the elapsed time between the initial experiment and our automated ranking as described here. In this case a like-for-like comparison was not possible.

\subsection{Procedure}

The study in which the manual rankings were generated is described in $\S 2.2$. Full methodology, materials, and instrumentation are available to allow third-parties to confirm our experiments at http://wel.cs. manchester.ac.uk/research/vicram/studies/metrics-study and as a technical report (Michailidou and Harper, 2006).

\subsection{Analysis plan}

The complexity score was generated based on the automated tool and not on a scale from 1-10. The data are ordinal (not interval) and therefore we ran non-parametric tests because we had a small sample size and non-normal data.

\subsection{Assumptions}

For easy presentation of the algorithm, we assume that the matrix and page have been extracted and all features of each block-level element have been converted into a value.

\subsection{Results}

In order to examine whether the ranking score that each page was assigned by the two different approaches (Manual and Computational evaluation) were related, we ran a bivariate correlation analysis for non-parametric data. Table 1 lists the ranking data that we used for the analysis. For each approach, a complexity average value was calculated and used to rank the pages in order. Figure 2 shows the relationship between the ranked data sets in a line graph where each line represents one data set.

We first compared the computational approach with the manual rankings. In this case, most of the stimuli received the same ranking, or one close to it. The Spearman correlation analysis showed that there is, indeed, a highly effective and significant positive relationship between the two ranking sets, $r_{s}=0.955$, $\mathrm{p}<0.001$ (see Table 1). The Kendall's correlation test between the two ranking scores reveals a positive

\footnotetext{
${ }^{15}$ http://wel.cs.manchester.ac.uk/research/vicram/studies/metrics-study/
} 
relationship between the manual ranking order and the computational ranking order, $\tau=0.841, \mathrm{p}<0.001$. Even if the Kendall correlation coefficient is smaller than the Spearman's it is still of large effect (anything of $>0.5$ has a large effect). Finally, running a Wilcoxon signed-ranked test shows that there are 11 ties, 6 computational ranking orders that are lower than the manual orders and two computational ranks that are higher than the manual rank. There was not a significant difference between the computational and manual orders $(z=-1.211, \mathrm{p}=0.226)$, which again shows that the two methodologies are significantly correlated.

From the line graph we can see the common ranks that the two evaluations produced as well as the misclassifications (see $\S 5$ ) that are still significantly close to each other. A correlation analysis was performed between the average complexity values calculated by the computational algorithm and the manual framework. The analysis showed that there is a significant correlation between the two sets, $\mathrm{r}_{s}=0.758$ and $\tau=$ 0.582 both with $p<0.001$. This is expected, since the ranks scored for each image were derived from these values.

Although there is a correlation between the file size rankings and the manual rankings, this is much smaller than the correlation between the computational and manual rankings for both the Spearman $\left(r_{s}=0.509\right.$, $\mathrm{p}=0.026)$ and Kendall $(\tau=0.322, \mathrm{p}=0.054)$ tests. The Wilcoxon signed-rank test shows that the two orders do not differ significantly $(z=-0.095, \mathrm{p}=0.924)$, but also that there are fewer matches between the ranks: only 2 are tied; 8 file size rankings are higher and 9 are lower.

Table 1: Complexity Mean Values and Ranking Order Using: Manual Card Sorting; Tuch filesize (Tuch et al., 2009); and Computational Tool. Ordered by 'Manual Rank' - where two Filesizes show the same $\mathrm{Kb}$ size the order is established using the exact byte Filesize.

\begin{tabular}{|l|r|r|r|r|r|r|r|r|}
\hline \hline Pages & PagelD & $\begin{array}{r}\text { Manual } \\
\text { Mean }\end{array}$ & $\begin{array}{r}\text { Manual } \\
\text { Mode }\end{array}$ & $\begin{array}{r}\text { Manual } \\
\text { Rank }\end{array}$ & $\begin{array}{r}\text { Tuch } \\
\text { Filesize }\end{array}$ & $\begin{array}{r}\text { Tuch } \\
\text { Rank }\end{array}$ & $\begin{array}{r}\text { Comp. } \\
\text { Mean }\end{array}$ & $\begin{array}{r}\text { Comp. } \\
\text { Rank }\end{array}$ \\
\hline \hline John Rylands Library & $\mathrm{S} 10$ & 3.75 & 01 & 01 & $231 \mathrm{~kb}$ & 12 & 0.067 & 01 \\
\hline MINT Group & $\mathrm{S} 03$ & 3.75 & 02 & 02 & $86 \mathrm{~kb}$ & 6 & 0.300 & 02 \\
\hline Peve Group & $\mathrm{S} 18$ & 8.17 & 04 & 03 & $53 \mathrm{~kb}$ & 3 & 0.440 & 03 \\
\hline Umber Group & $\mathrm{S} 12$ & 6.17 & 05 & 04 & $201 \mathrm{~kb}$ & 11 & 0.667 & 07 \\
\hline Vodafone UK & $\mathrm{S} 15$ & 4.92 & 06 & 05 & $94 \mathrm{~kb}$ & 8 & 0.680 & 05 \\
\hline Informatics Process Gp & $\mathrm{S} 08$ & 7.08 & 06 & 06 & $53 \mathrm{~kb}$ & 2 & 0.925 & 06 \\
\hline John Rylands Library Ctlg & $\mathrm{S} 17$ & 8.33 & 07 & 07 & $37 \mathrm{~kb}$ & 1 & 0.520 & 04 \\
\hline Google & $\mathrm{S} 09$ & 6.17 & 08 & 08 & $66 \mathrm{~kb}$ & 4 & 0.633 & 05 \\
\hline Dr. Stevens Robert & $\mathrm{S} 01$ & 9.00 & 09 & 09 & $94 \mathrm{~kb}$ & 7 & 0.633 & 09 \\
\hline Dr. Harper Simon & $\mathrm{S} 14$ & 11.67 & 11 & 10 & $262 \mathrm{~kb}$ & 15 & 0.667 & 08 \\
\hline National Rail & $\mathrm{S} 19$ & 7.75 & 12 & 11 & $389 \mathrm{~kb}$ & 17 & 0.714 & 10 \\
\hline Yahoo & $\mathrm{S} 07$ & 10.75 & 13 & 12 & $319 \mathrm{~kb}$ & 16 & 1.900 & 14 \\
\hline Geneontology & $\mathrm{S} 20$ & 14.67 & 16 & 13 & $82 \mathrm{~kb}$ & 5 & 1.930 & 13 \\
\hline BBC UK & $\mathrm{S} 04$ & 16.25 & 16 & 14 & $172 \mathrm{~kb}$ & 10 & 1.077 & 12 \\
\hline Amazon & $\mathrm{S} 05$ & 13.50 & 17 & 15 & $459 \mathrm{~kb}$ & 19 & 2.075 & 15 \\
\hline Computer Science MCR & $\mathrm{S} 06$ & 14.75 & 18 & 16 & $246 \mathrm{~kb}$ & 14 & 0.691 & 16 \\
\hline MSN & $\mathrm{S} 16$ & 15.58 & 18 & 17 & $242 \mathrm{~kb}$ & 13 & 2.080 & 17 \\
\hline Manchester Online & $\mathrm{S} 13$ & 16.25 & 19 & 18 & $401 \mathrm{~kb}$ & 18 & 3.655 & 18 \\
\hline Tesco & $\mathrm{S} 11$ & 17.00 & 20 & 19 & $147 \mathrm{~kb}$ & 9 & 2.071 & 16 \\
\hline \hline
\end{tabular}

Remember that if our hypothesis holds (based on (Yesilada et al., 2008)) - that visual complexity, expressed only as block-level elements within the DOM - is an indicator of perceived complexity, then the manual ranking places pages with low perceived complexity to the left and those with high perceived complexity to the right. If our algorithm follows the manual sequence, then we can state that we can programmatically identify the perceived complexity of Web pages. The overall results suggest that the conceptual model and prototype introduced are both promising and useful. While there are inconsistencies between the programmatic and manual classifications, these can be explained in terms of the other criteria used by humans for classification, such as words per block level element, colour, and image and animation size and quantity. However, the programmatic distribution did show that, with some minor refinements to the algorithm, an accuracy of over $86 \%$ can be achieved. Indeed, plotting the misclassification error reveals that $90 \%$ of 
sample Web pages are correctly classified into simple, medium and complex. While, with the programatic algorithm, dedicated ranking or comparing is difficult, visually simple and complex pages can be recognised and pointed out following the appropriate classification. In this case:

The results from our initial algorithm are encouraging. While block-level structure alone is not an absolute guide to user perception, it does allow us to predict based on a statistically significant and strong correlation; and therefore seems to be a good proxy indicator of perceived visual complexity.

Indeed, this work has allowed us to understand why this is the case and enabled us to make changes that increase the ability to predict this complexity. This said, it is still worth considering that we can (without any modifications) predict visual complexity perception, to a high degree of accuracy.

\section{Analysis / Critique}

The manual rankings are highly and significantly correlated with the rankings produced by the computational algorithm, and match these much better than they do the rankings produced by considering image file size. However, we can see from our results that we have a number of mis-classified / sequenced Web pages when compared to our manual sequence. These are due to three generalizable reasons: Invisible DIV Elements; Non-Structural Factors; and Errors in the XHTML Code. (1) Invisible DIV Elements: this case is due to the simplicity of our algorithm when compared with human perception. Our algorithm does not take into account the background colour of each block-level element. In this case certain pages have a number of nested 'DIV' elements all invisible to the human user. Therefore, programmatic classifications tend toward complex where as human ones tend toward simple. We can address this problem by querying each DIV element in the cascading style sheet. (2) Non-Structural Factors: contrary to our last case, the grid algorithm predicts simplicity but the manual classification suggests complexity. The main reasons for this

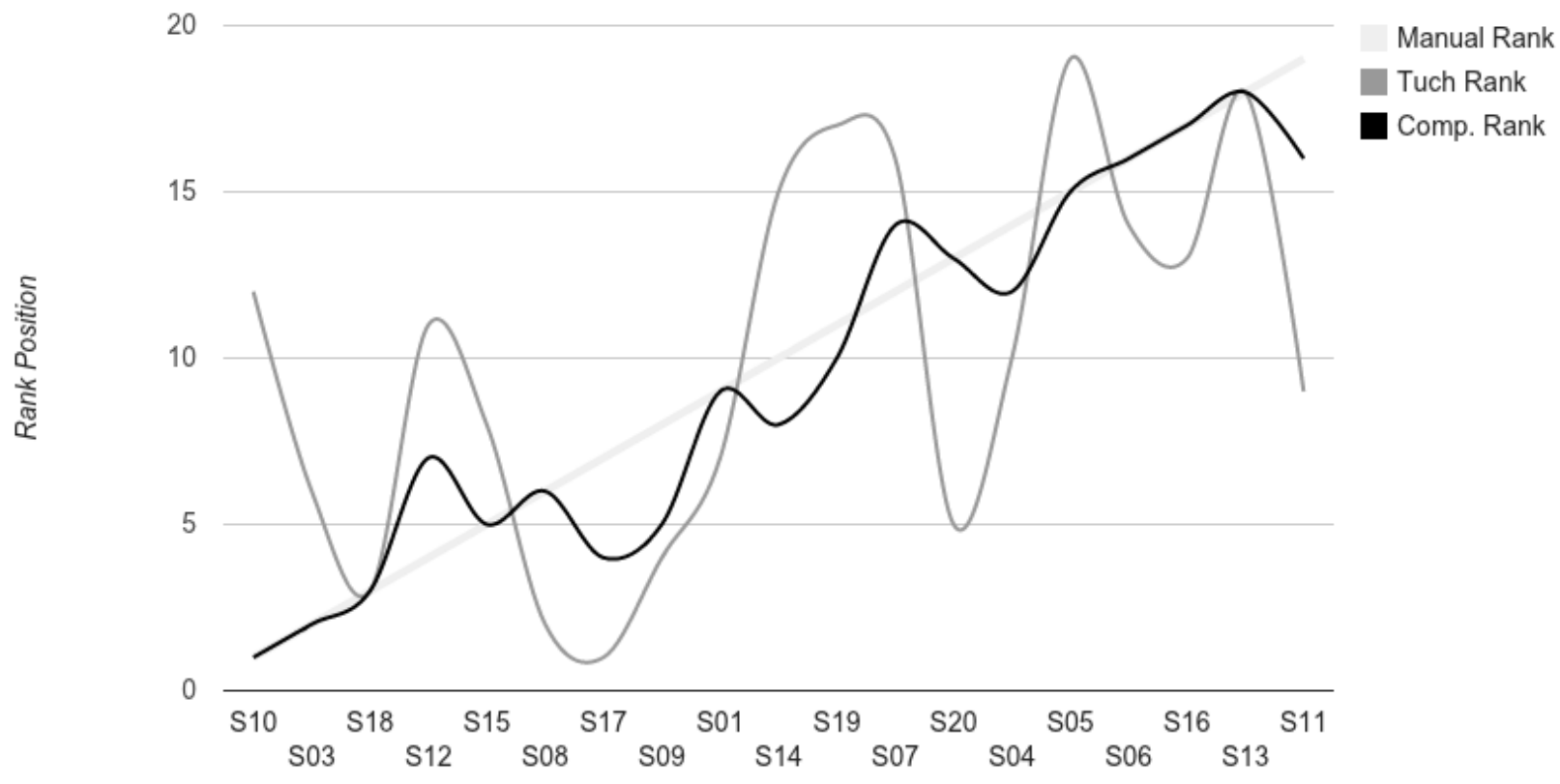

Web Sites

Figure 2: Ranking Scores from Manual and Computational Algorithm - Line 
anomaly include: (2a) The font style is difficult to read (such as 'Brush Script MT'); (2b) Line lengths are too long when reading the content; and (2c) the colour scheme has a high degree of contrast. (3) Errors in the XHTML Code: our third and final failure is due to designer bad practice such as hidden layout and errors in the XHTML code such as extra null $<$ p $>$ elements, without a closing tag, to add white space. In this case a change in the XHTML renderer or an enhancement for the HTML-Tidy software would remove this failure.

In summary, most of the algorithm's misclassifications are caused by the differences between the code and the visual rendering of the page; the conflict between semantic structure and syntactic structure. The layout of a Web page, the use of various fonts, colours, images, and other visual cues convey navigational and semantic information to users. Unfortunately these visual cues are not accessible from the source code or DOM. However there are some further refinements to the algorithm which are needed to make it more effective at the application level (post-experimentation):

1. Attributes such as screen size have been predefined, and the width and height of Web pages are calculated using these pre-defined averages. Because the presentation of a Web page depends on user agents' features (e.g., screen size and Web browser setting) we could change the algorithm to increase the algorithms flexibility, but this makes comparison between pages harder. We therefore need to balance accuracy of the analysis with accuracy when undergoing comparison.

2. Embedded block-level elements can be counted according to their hierarchy level (denoted by W). Normally parent blocks should be weighted higher than their children.

3. The sum of average and average deviation can be changed to sum weight factors:

$$
S u m=W 1 \times a v e+W 2 \times d e v
$$

This is because the deviation has more of an effect on visual complexity than average density. We can therefore adjust W2 to be larger than W1 (see item 2 above).

Further, our lower and higher ranks show us potential improvements and future work for both our algorithm and the tools used in the creation of the experimental test harnesses. Currently our framework is reliant on third party software such as the DOM parser implemented by the W3C DOM parser API. It is possible that some DOM3 specifications are not supported ${ }^{16}$. More importantly the rendering result is created from the Flying Saucer XHTML renderer and is not as precise as popular browsers such as Gecko-JRex. These technical improvements are useful for ensuring the longer term flexibility of a development application and their addition would not have any real affect on the results or the accuracy of the ranking grid algorithm.

Finally, it may seem that our initial formative work was subjective, qualitative, and had too few participants $^{17}$ for any general conclusions to be drawn (Harper et al., 2009). In some regard we would agree with these criticisms but feel that once the context of the entire programme of work is understood, these are mitigated. Initially, we wanted to understand perceptions of visual complexity, i.e. what makes users navigate away from a page without even investigating it further. As part of our preliminary investigation we began to realise it was the visual look, and the interactive complexity that this look and feel implied. Along with the collection of extensive qualitative data we decided to ask users to rank Web pages based on their subjective perceptions of complexity so we could understand this area further. We soon discovered that Web pages ranked closely to each other had similar visual traits, and that these traits could be identified programmatically. This lead us to create our algorithm and experimental analysis tool, as described here. To confirm the generalizability of the algorithm it is necessary to test it with further, live, Web pages, ranked by different participants. In addition, it would be useful to examine how demographic factors like age, gender, and culture affect user rankings; there is evidence, for example, that symmetry has a favourable effect on men's aesthetic judgements of Web pages, but no effect on women's (Tuch et al., 2010). The algorithm was designed to work with 'standard' Web pages between $760 \& 990$ pixels wide. It would be interesting to examine systematically how the algorithm works for screens of different sizes or with different resolutions. In this case, we are not stating that the algorithm is infallible or that improvements cannot be made; what we are asserting is that our initial results show promise.

\footnotetext{
${ }^{16}$ Xerces Web site http://www.apache.org/xerces

${ }^{17}$ Although we match Pandir and Knights (Pandir and Knight, 2006) 12 participants and exceeded their Web site sample by 8.
} 


\section{Conclusions}

Our experiment was an investigation of how structural elements can predict the level of perceived visual complexity of a Web page. The evaluation showed that the number of block-level components is positively related to the perceived visual complexity of the page. We have demonstrated the ability to automatically classify Web page complexity, to an average accuracy of $86 \%$, with regard to block-level elements. We now continue this work by building a framework based on the density and diversity of Web page structural elements that provide a visual complexity score prediction. The framework will be implemented into the ACTF Eclipse framework, as an extension of the aDesigner software, and will automatically analyse a Web page with respect to its perceived visual complexity. For each Web page, users and designers will be provided with a score that determines the page's level of perceived visual complexity and an overlay 'heatmap' that notes the areas that will be perceived as most visually complex. We then propose a final user evaluation to examine the success level of the tool. During an online experiment, users will be asked to rank Web pages based on their perceived visual complexity. These rankings will be compared with the rankings that the algorithm returned for the same pages. We predict results showing a strong and significant correlation between users' visual complexity perception of a Web page and the algorithm prediction rankings. This will demonstrate that the perceived visual complexity level of Web pages can be automatically predicted up to a certain ratio based on the structural elements of the page. The tool could therefore be used to gain an initial perception of the visual layout of the page, and by designers to improve their designs towards balancing Web page visual complexity with usability, aesthetics and accessibility. Investigating users' visual perception and Web interaction will enable us to understand the linkage between visual complexity, cognition and Web page layout. This research may demonstrate that an initial step into enhancing accessibility for visually impaired users can be made through understanding sighted users' perception (Brown et al., 2012b).

\subsection{Onward...}

In the future we will begin to make some leaps into the visual disability space, by associating perceived visual complexity with metrics for understanding accessibility. Indeed, our previous work has already demonstrated that there is a relationship between visual complexity and perceived complexity and that a visually complex page is just as complex to users without the ability to see the content be they visually disabled (Harper et al., 2009) or situationally impaired (Sears et al., 2003).

The information on most of these Web pages is visually fragmented and organised into groups (Asakawa and Takagi, 2000). In this way, when sighted users reach a Web page, they can scan the page and obtain a comprehension of it in few seconds. By that time they have decided if the page is relevant to their task and have moved towards the part of the page that interests them. We wish to understand what happens when visually disabled people try to interact with such resources. Problems arise because the implicit visual cues presented on a Web page cannot be accessed and used by people with disabilities, especially those who are visually disabled (Plessers et al., 2005). The increased visual complexity of a Web page is described through Web site accessibility and usability frameworks; we will attempt to relate the visual complexity of a Web page to users' interaction patterns in order to aid visually disabled users.

\section{Acknowledgements}

This work was undertaken as part of the ViCRAM project, conducted in the Web Ergonomics Lab in the School of Computer Science at the University of Manchester (UK).

\section{References}

A. Holzinger and, M. K.-R. and Albert, D. (2008). Dynamic media in computer science education; content complexity and learning performance: Is less more? Educational Technology \& Society, 11(1):279-290.

Altaboli, A. and Lin, Y. (2011). Investigating effects of screen layout elements on interface and screen design aesthetics. Adv. in Hum.-Comp. Int., 2011:5:1-5:10.

Amitay, E., Carmel, D., Darlow, A., Lempel, R., and Soffer, A. (2003). The connectivity sonar: detecting 
site functionality by structural patterns. In HYPERTEXT '03: Proceedings of the fourteenth ACM conference on Hypertext and hypermedia, pages 38-47, New York, NY, USA. ACM Press.

Asakawa, C. and Takagi, H. (2000). Annotation-based transcoding for nonvisual web access. In Assets '00: Proceedings of the fourth international ACM conference on Assistive technologies, pages 172-179, New York, NY, USA. ACM Press.

Bar, M., Neta, M., and Linz, H. (2006). Very first impressions. Emotion, 6(2):269-278.

Berlyne, D. (1960). Conflict, arousal and curiosity. New York: MCGraw-Hill.

Berlyne, D. (1974). Studies in the new experimental aesthetics. Washington DC: Hemisphere.

Brown, A., Jay, C., Chen, A., and Harper, S. (2012a). The uptake of web 2.0 technologies, and its impact on visually disabled users. Universal Access in the Information Society, 11(3):185-199.

Brown, A., Jay, C., and Harper, S. (2012b). Tailored presentation of dynamic web content for audio browsers. Int. J. Hum.-Comput. Stud., 70(3):179-196.

Choi, J. H. and Lee, H.-J. (2012). Facets of simplicity for the smartphone interface: A structural model. Int. J. Hum.-Comput. Stud., 70(2):129-142.

Donderi, D. C. (2006). An information theory analysis of visual complexity and dissimilarity. Perception, 35(6):823-835.

Faraday, P. and Sutcliffe, A. (1998). Making contact points between text and images. In MULTIMEDIA '98: Proceedings of the sixth ACM international conference on Multimedia, pages 29-37, New York, NY, USA. ACM Press.

Germonprez, M. and Zigurs, I. (2003). Causal factors for web site complexity. Working Papers on Information Environments, Systems and Organizations, (3).

Harper, S. and Bechhofer, S. (2007). Sadie: Structural semantics for accessibility and device independence. ACM Trans. Comput.-Hum. Interact., 14(2):10.

Harper, S., Michailidou, E., and Stevens, R. (2009). Toward a definition of visual complexity as an implicit measure of cognitive load. ACM Trans. Appl. Percept., 6(2):1-18.

Harper, S. and Yesilada, Y. (2010). Emerging technologies and web accessibility: research challenges and opportunities focussing on vision issues. Disability \& Rehabilitation: Assistive Technology, pages 1-19.

Heaps, C. and Handel, C. H. (1999). Similarity and features of natural textures. Journal of Experimental Psychology: Human Perception and Performance, 25:299-320.

Hoffos, S. and Sharpless, G. (1991). The CD-I designers guide. McGraw-Hill.

Holzinger, A., Stickel, C., Fassold, M., and Ebner, M. (2009). Seeing the system through the end users' eyes: Shadow expert technique for evaluating the consistency of a learning management system. In Proceedings of the 5th Symposium of the Workgroup Human-Computer Interaction and Usability Engineering of the Austrian Computer Society on $\mathrm{HCl}$ and Usability for e-Inclusion, USAB '09, pages 178-192, Berlin, Heidelberg. Springer-Verlag.

Ivory, M. Y. and Megraw, R. (2005). Evolution of web site design patterns. ACM Trans. Inf. Syst., 23(4):463-497.

Ivory, M. Y., Sinha, R. R., and Hearst, M. A. (2000). Preliminary findings on quantitative measures for distinguishing highly rated information-centric web pages. In Proceedings of 6th Conference on Human Factors and the Web. 
Ivory, M. Y., Sinha, R. R., and Hearst, M. A. (2001). Empirically validated web page design metrics. In $\mathrm{CHI}$ '01: Proceedings of the SIGCHI conference on Human factors in computing systems, pages 53-60, New York, NY, USA. ACM Press.

Jay, C., Stevens, R., Glencross, M., and Chalmers, A. (2006). How people use presentation to search for a link: expanding the understanding of accessibility on the web. In W4A: Proceedings of the 2006 international cross- disciplinary workshop on Web accessibility (W4A), pages 113-120, New York, NY, USA. ACM Press.

Lazar, J., Feng, J., and Allen, A. (2006). Determining the impact of computer frustration on the mood of blind users browsing the web. In Assets '06: Proceedings of the 8th international ACM SIGACCESS conference on Computers and accessibility, pages 149-156, New York, NY, USA. ACM Press.

Leder, H., Belke, B., Oeberst, A., and Augustin, D. (2004). A model of aesthetic appreciation and aesthetic judgments. British Journal of Psychology, 1995(4):489-508.

Lindgaard, G., Fernandes, G., Dudek, C., and Brown, J. (2006). Attention web designers: You have 50 milliseconds to make a good first impression! Behaviour and Information Technology. Taylor and Francis, Vol. 25, No. 2:115-126.

Merriam-Webster (2006). Merriam-webster online dictionary. (Accessed January 30, 2006).

Michailidou, E. (2005). Metrics of visual complexity. Master's thesis, Computer Science.

Michailidou, E. and Harper, S. (2006). Vicram: Visual complexity rankings and accessibility metrics. Technical report (http://hcw-eprints.cs.man.ac.uk/25/).

Moshagen, M. and Thielsch, M. (2010). Facets of visual aesthetics. Int. J. Hum.-Comput. Stud., 68(10):689-709.

Oliva, A., Mack, M. L., Shrestha, M., and Peeper, A. (2004). Identifying the perceptual dimensions of visual complexity of scenes. In Proceedings of the 26th Annual Meeting of the Cognitive Science Society.

Pandir, M. and Knight, J. (2006). Homepage aesthetics: The search for preference factors and the challenges of subjectivity. Interact. Comput., 18:1351-1370.

Plessers, P., Casteleyn, S., Yesilada, Y., Troyer, O. D., Stevens, R., Harper, S., and Goble, C. (2005). Accessibility: a web engineering approach. In WWW '05: Proceedings of the 14th international conference on World Wide Web, pages 353-362, New York, NY, USA. ACM Press.

Rayner, K. (1998). Eye movements in reading and information processing: 20 years of research. 124(3):372-422.

Reber, R., Schwarz, N., and Winkielman, P. (2004). Processing fluency and aesthetic pleasure: is beauty in the perceiver's processing experience? Pers Soc Psychol Rev, 8(4):364-382.

Sears, A., Lin, M., Jacko, J., and Xiao, Y. (2003). When Computers Fade...Pervasive Computing and Situationally-Induc pages 1298-1302. Lawrence Erlbaum Associates, Hillsdale.

Stickel, C., Ebner, M., and Holzinger, A. (2010). The xaos metric: understanding visual complexity as measure of usability. In Proceedings of the 6th international conference on $\mathrm{HCl}$ in work and learning, life and leisure: workgroup human-computer interaction and usability engineering, USAB'10, pages 278-290, Berlin, Heidelberg. Springer-Verlag.

Takagi, H., Asakawa, C., Fukuda, K., and Maeda, J. (2004). Accessibility designer: visualizing usability for the blind. In Assets '04: Proceedings of the 6th international ACM SIGACCESS conference on Computers and accessibility, pages 177-184, New York, NY, USA. ACM Press.

Thielsch, M. and Hirschfeld, G. (2012). Spatial frequencies in aesthetic website evaluations - explaining how ultra-rapid evaluations are formed. Ergonomics, page ePub ahead of print. 
Tuch, A., Kreibig, S., Roth, S., Bargas-Avila, J., Opwis, K., and Wilhelm, F. (2011). The role of visual complexity in affective reactions to webpages: Subjective, eye movement, and cardiovascular responses. Affective Computing, IEEE Transactions on, 2(4):230 -236.

Tuch, A. N., Bargas-Avila, J. A., and Opwis, K. (2010). Symmetry and aesthetics in website design: It's a man's business. Computers in Human Behavior, 26:1831-1837.

Tuch, A. N., Bargas-Avila, J. A., Opwis, K., and Wilhelm, F. H. (2009). Visual complexity of websites: Effects on users' experience, physiology, performance, and memory. International Journal of Human-Computer Studies, 67(9):703 - 715.

Yesilada, Y., Jay, C., Stevens, R., and Harper, S. (2008). Validating the use and role of visual elements of web pages in navigation with an eye-tracking study. In WWW '08: Proceeding of the 17th international conference on World Wide Web, pages 11-20, New York, NY, USA. ACM. 